\title{
TITLE:
}

\section{Criterion of mechanical instabilities for dislocation structures}

\section{AUTHOR(S):}

Yan, Yabin; Kondo, Tadahiro; Shimada, Takahiro; Sumigawa, Takashi; Kitamura, Takayuki

\section{CITATION:}

Yan, Yabin ...[et al]. Criterion of mechanical instabilities for dislocation structures. Materials Science and Engineering: A 2012, 534: 681-687

ISSUE DATE:

2012-02

URL:

http://hdl.handle.net/2433/153280

\section{RIGHT:}

(C) 2011 Elsevier B.V.; This is not the published version. Please cite only the published version.; この論文は出版社版でありません。引用の際に は出版社版をご確認ご利用ください。 


\title{
Criterion of Mechanical Instabilities for Dislocation Structures
}

\author{
Yabin Yan*, Tadahiro Kondo, Takahiro Shimada, Takashi Sumigawa, Takayuki Kitamura \\ Department of Mechanical Engineering and Science, Graduate School of Engineering, \\ Kyoto University, Yoshida-honmachi, Sakyo-ku, Kyoto 606-8501, Japan
}

\begin{abstract}
To understand the nature of mechanical instabilities of dislocation structures, which plays a central role, for example, in determining the plastic behavior and fatigue in crystalline metals, it is essential to investigate a critical condition in which a dislocation structure collapses. A criterion for the mechanical instability of arbitrary dislocation structures is proposed in this paper. According to the criterion, the mechanical instability can be described by the positiveness of the minimum eigenvalue of the Hessian matrix, which is composed by the second-order differential of potential energy of the system with respect to the dislocation coordinates. In addition, the collapse mode can be simultaneously determined by the eigenvector of the minimum eigenvalue. We applied the proposed criterion to the veins and dislocation walls under external loading, and it successfully describes the onset of instabilities and the corresponding collapse modes, regardless of the difference in structures and sizes. This success in the criterion paves the way to address the mechanical instability issues on more complex dislocation structures.
\end{abstract}

Keywords: Criterion; Instability; Dislocation structure; Vein; Dislocation Wall; Deformation mode; Dislocation theory

\footnotetext{
${ }^{*}$ Corresponding author. Tel: +81 75753 5192; fax: +81 757535256 .

E-mail address: yan.yabin@ht5.ecs.kyoto-u.ac.jp (Y. Yan).
} 


\section{Introduction}

Dislocations, which are one-dimensional topological defects, are central to understand the mechanical properties of crystalline materials. The motion and interaction of dislocations over mesoscopic distances are fundamental to many phenomena in crystalline materials, such as plastic deformation and fracture of bulk solids. For example, strain hardening is induced in crystalline materials by the increased number of interacting dislocations that trap each other in minimum energy configurations [1], and the soft persistent slip bands (PSBs) cause fatigue cracking due to the localization of plastic strain [2,3]. On the other hand, dislocations in deformed metals tend to cluster into various kinds of dense regions with high dislocation densities, which are separated by regions with reduced dislocation densities, resulting in the formation of characteristic dislocation structures [4,5] such as dislocation cells, slip bands, microshear bands, PSBs, and dislocation tangles [6-12]. In addition, the configuration of dislocation structures changes with the motion and multiplication of dislocations due to applied external load. For example, dislocation structures in pure metals change from veins to PSBs, and further cell or labyrinth structures with the development of fatigue [13-18]. This indicates that dislocation structure evolution dominates typical plastic deformation and fracture behavior in fatigue of metal. It is thus critically important to investigate the instability of dislocation structures to gain a microscopic understanding of the deformation and fracture characteristics of metals. However, due to the complexity of numerous dislocations included in a structure, the mechanism of instability of dislocation structures has rarely been investigated in detail. It is thus necessary to develop an appropriate criterion to precisely evaluate the mechanical instability of dislocation structures.

In this study, a criterion is proposed to describe the mechanical instability of arbitrary dislocation structures from the viewpoint of energy balance to external load by 
considering all the degrees of freedom of dislocations. The proposed criterion is applied to dislocation structures such as veins and dislocation walls subjected to external loads.

\section{Analytical instability criterion for dislocation structures}

For simplicity, we consider a dislocation structure on the primary slip plane of (111) of a face-centered cubic (fcc) metal crystal under a constant external load, where the $x$-axis corresponds to the primary slip direction of [101] and the $y$-axis is aligned with the [111] direction of the crystal. For further condition, e.g., the multi-slip condition, the same formulation is available by appropriately accounting for the degrees of freedom of the system.

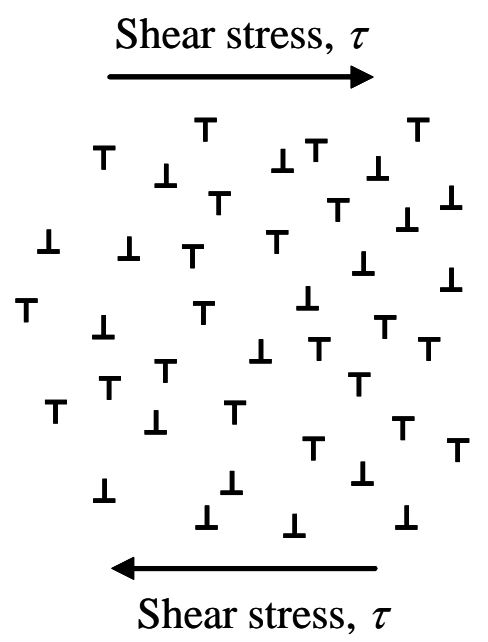

Fig. 1 Schematic illustration of a two-dimensional dislocation structure under a shear stress, $\tau$, due to an external load.

We consider a two-dimensional dislocation structure consisting of $N$ parallel edge dislocations under an external load, as schematically shown in Fig. 1. The edge dislocations are numbered from 1 to $N$ and the coordinates and the Burgers vector of the $i$-th dislocation are $\boldsymbol{r}_{i}={ }^{t}\left(x_{i}, y_{i}\right)$ and

$$
\boldsymbol{b}_{i}=\left(\zeta_{i} b, 0,0\right) \quad\left(\zeta_{i}= \pm 1\right),
$$


respectively. Here, $b$ is the magnitude of the Burgers vector, and $\zeta_{\mathrm{i}}$ represent the positive $(\perp)$ and negative $(T)$ edge dislocations. Since the dislocation motion is restrained in the direction of the Burgers vector (the $x$-direction), the dislocation structure has $N$ degrees of freedom. Moreover, to eliminate rigid translation of the system, the system has a total of $M=N-1$ degrees of freedom. Therefore, an arbitrary change to the configuration of the dislocation structure can be described by an $M$-dimensional vector consisting of the $x$-coordinates of the dislocations,

$$
\boldsymbol{X} \equiv{ }^{t}\left(x_{2}, x_{3}, \cdots, x_{N}\right)={ }^{t}\left(X_{1}, X_{2}, \cdots, X_{M}\right) .
$$

The total energy of the system, $\Pi$, in an equilibrium state $\left(\boldsymbol{X}_{0}\right)$ consists of the interaction potential energy of dislocations $U$, the work done by the external load $W$, and the dislocation self-energy $E_{\text {self }}$,

$$
\Pi=U+W+E_{\text {self }},
$$

where $U$ is the sum of the interaction potential energy originated by every two dislocations and is given by

$$
U=\left.\frac{1}{2} \sum_{i=1}^{N} \sum_{j=1, j \neq i}^{N} \frac{G \zeta_{i} \zeta_{j} b^{2}}{2 \pi(1-v)}\right|_{\{} \log \frac{\sqrt{\left(x_{i}-x_{j}\right)^{2}+\left(y_{i}-y_{j}\right)^{2}}}{R_{\mathrm{c}}}+\left.\frac{\left(y_{i}-y_{j}\right)^{2}}{\left(x_{i}-x_{j}\right)^{2}+\left(y_{i}-y_{j}\right)^{2}}\right|_{j}
$$

$W$ is

$$
W=\sum_{i=1}^{N} \zeta_{i} b \int \tau_{y x} d x_{i}
$$

$E_{\text {self }}$ has the form [19]

$$
E_{\text {self }}=N \alpha G b^{2} \quad(\alpha \square 0.4) .
$$

Here, $G$ is the shear modulus, $v$ is Poisson's ratio, $R_{\mathrm{c}}$ is the cut-off radius of the integration path, and $\tau_{x y}$ is the shear stress component imposed by an external load. The Taylor's series expansion of $\Pi\left(\boldsymbol{X}_{0}\right)$ in terms of an infinitesimal displacement, $\Delta \boldsymbol{X}$, has the form 


$$
\begin{aligned}
\Pi\left(\boldsymbol{X}_{0}+\Delta \boldsymbol{X}\right)= & \Pi\left(\boldsymbol{X}_{0}\right)+\sum_{i=1}^{M}\left(\left.\frac{\partial U}{\partial X_{i}}\right|_{X=X_{0}}+\left.\frac{\partial W}{\partial X_{i}}\right|_{X=X_{0}}\right) \Delta X_{i} \\
& +\left.\frac{1}{2} \sum_{i=1}^{M} \sum_{j=1}^{M} \frac{\partial^{2} U}{\partial X_{i} \partial X_{j}}\right|_{X=X_{0}} \Delta X_{i} \Delta X_{j}+\cdots, \\
& +\left.\frac{1}{2} \sum_{i=1}^{M} \sum_{j=1}^{M} \frac{\partial^{2} W}{\partial X_{i} \partial X_{j}}\right|_{X=X_{0}} \Delta X_{i} \Delta X_{j}+\cdots .
\end{aligned}
$$

Because the system is at equilibrium, the second term on the right-hand side is zero. Additionally, since the external load is constant, we obtain

$$
\left.\frac{\partial^{2} W}{\partial X_{i} \partial X_{j}}\right|_{X=X_{0}}=0 \quad(i, j=1, \cdots, M) .
$$

By neglecting higher-order terms, the change in the total energy, $\Delta \Pi$, induced by the infinitesimal displacement, $\Delta \boldsymbol{X}$, is written as

$$
\Delta \Pi=\Pi\left(\boldsymbol{X}_{0}+\Delta \boldsymbol{X}\right)-\Pi\left(\boldsymbol{X}_{0}\right)=\frac{1}{2}^{t} \Delta \boldsymbol{X} \mathbf{H} \Delta \boldsymbol{X},
$$

where $\mathbf{H}$ is the $M \times M$ Hessian matrix with components, $H_{i j}$,

$$
\left.H_{i j} \equiv \frac{\partial^{2} U}{\partial X_{i} \partial X_{j}}\right|_{X=X_{0}} \quad(i, j=1, \cdots, M) .
$$

Substituting Eq. (4) into Eq. (10), the components of $\mathbf{H}$ are obtained as

$$
\frac{\partial^{2} U}{\partial x_{i} \partial x_{j}}=\left\{\begin{array}{l}
\frac{G \zeta_{i} b^{2}}{2 \pi(1-v)} \times \sum_{k \neq i} \zeta_{k} \frac{\left(x_{i}-x_{k}\right)^{4}-6\left(x_{i}-x_{k}\right)^{2}\left(y_{i}-y_{k}\right)^{2}+\left(y_{i}-y_{k}\right)^{4}}{\left\{\left(x_{i}-x_{k}\right)^{2}+\left(y_{i}-y_{k}\right)^{2}\right\}^{3}} \\
-\frac{G \zeta_{i} \zeta_{j} b^{2}}{2 \pi(1-v)} \times \frac{\left(x_{i}-x_{j}\right)^{4}-6\left(x_{i}-x_{j}\right)^{2}\left(y_{i}-y_{j}\right)^{2}+\left(y_{i}-y_{j}\right)^{4}}{\left\{\left(x_{i}-x_{j}\right)^{2}+\left(y_{i}-y_{j}\right)^{2}\right\}^{3}}
\end{array} \quad(i \neq j) .\right.
$$

By solving the eigenvalue problem of the Hessian matrix $\mathbf{H}$,

$$
\mathbf{H} \boldsymbol{p}_{i}=\eta_{i} \boldsymbol{p}_{i} \quad(i=1, \cdots, M),
$$

the eigenvalues $\eta_{i}\left(\eta_{1} \leq \cdots \leq \eta_{i} \leq \cdots \leq \eta_{M}\right)$ and the corresponding eigenvectors $\boldsymbol{p}_{i}$ are obtained. Using the eigenvectors $\boldsymbol{p}_{i}$, the matrix $\mathbf{H}$ is diagonalized as 


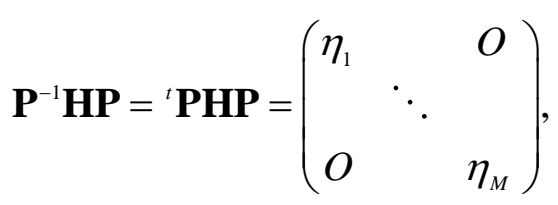

where $\mathbf{P}=\left(\boldsymbol{p}_{1} \cdots \boldsymbol{p}_{M}\right)$. Since the eigenvectors of the Hessian matrix are related by

$$
\boldsymbol{p}_{i} \cdot \boldsymbol{p}_{j}=\delta_{i j}
$$

where $\delta_{i j}$ is the Kronecker delta, the set of eigenvectors $\left\{\boldsymbol{p}_{i}\right\}$ is an orthogonal base of $M$-dimensional vector space. Thus, the infinitesimal displacement, $\Delta \boldsymbol{X}$, can be expressed as a linear combination of the eigenvectors $\boldsymbol{p}_{i}$ as

$$
\Delta \boldsymbol{X}=\sum_{i=1}^{M} u_{i} \boldsymbol{p}_{i}=\boldsymbol{P} \boldsymbol{u},
$$

where $u_{i}$ is the component of $\Delta \boldsymbol{X}$ in the $\boldsymbol{p}_{i}$ direction and $\boldsymbol{u}={ }^{t}\left(u_{1}, \cdots, u_{M}\right)$. Therefore, the change in the total energy, $\Delta \Pi$, due to the infinitesimal displacement, $\Delta \boldsymbol{X}$, is written as

$$
\Delta \Pi=\frac{1}{2}{ }^{t}(\mathbf{P u}) \mathbf{H}(\mathbf{P u})=\frac{1}{2} \boldsymbol{u}\left({ }^{t} \mathbf{P H P}\right) \boldsymbol{u}=\frac{1}{2} \sum_{i=1}^{M} \eta_{i} u_{i}^{2} .
$$

When the change in the total energy $\Delta \Pi$ for the infinitesimal displacement $\Delta \boldsymbol{X}$ is positive, the dislocation structure is stable and all the eigenvalues are positive (see Eq. (16)). On the other hand, when $\Delta \Pi$ is negative, the dislocation structure is unstable. Since $\eta_{1} \leq \cdots \leq \eta_{i} \leq \cdots \leq \eta_{M}$, the critical condition $\Delta \Pi=0$ appears when the smallest eigenvalue reaches zero

$$
\begin{gathered}
\eta_{1}=0, \\
u_{i}=0 \quad(i=2, \cdots, M) .
\end{gathered}
$$

Under this condition, $\Delta \boldsymbol{X}$ becomes

$$
\Delta \boldsymbol{X}=\mathbf{P u}=u_{1} \boldsymbol{p}_{1} .
$$

Here, $u_{1}$ has an arbitrary magnitude and $\boldsymbol{p}_{1}$ is determined by the Hessian matrix $\mathbf{H}$.

Eq. (17) is the criterion for the instability of a dislocation structure. Equation (19) shows that the dislocation displacement $\Delta X$ at the onset of instability is proportional to the eigenvector, $\boldsymbol{p}_{1}$. Thus, the displacement mode at the collapse of the dislocation 
structure is described by the eigenvector $\boldsymbol{p}_{1}$.

The above formulation can be extended to three-dimensional systems by adding the corresponding degree of freedom (displacement along the direction of the Burgers vector) of discrete dislocation points to the freedom vector $\boldsymbol{X}$ in Eq. (2).

\section{Instability of dislocation structures due to an external load}

\subsection{Simulation models}

Simulation models of veins and dislocation walls in Taylor-Nabarro lattices [20,21] are employed here because of their importance in the fatigue process. Since the purpose of this study is to evaluate the proposed criterion, ideal veins and dislocation walls are used, although the scale and arrangement of dislocations are quite different from actual dislocation structures. 


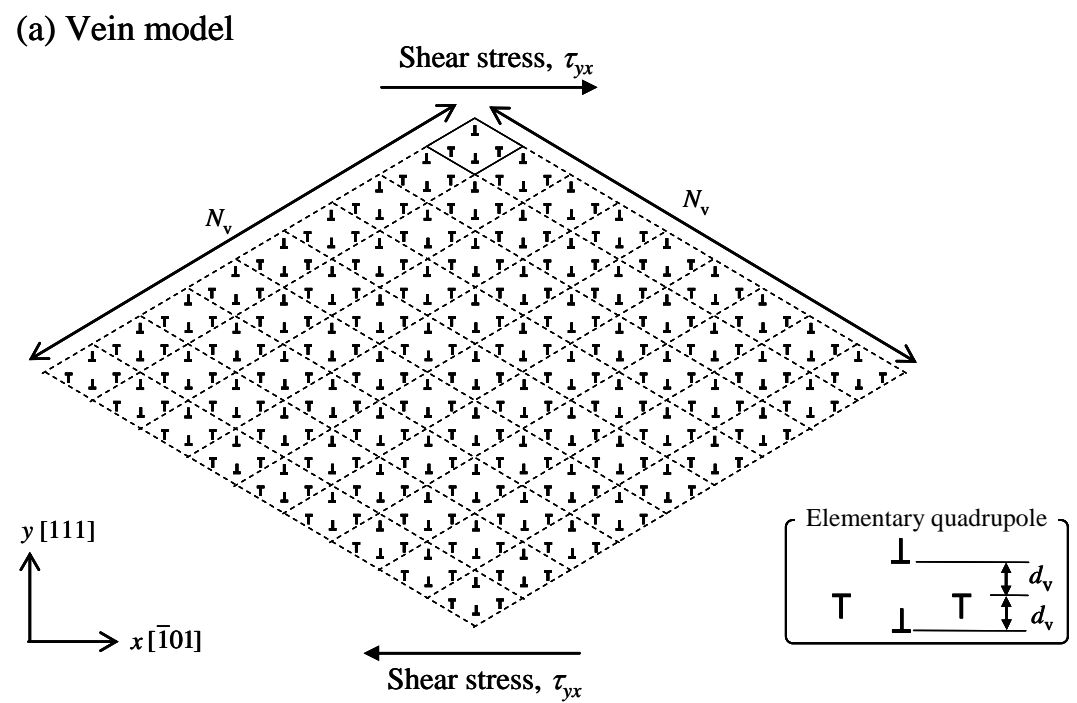

(b) Dislocation wall model
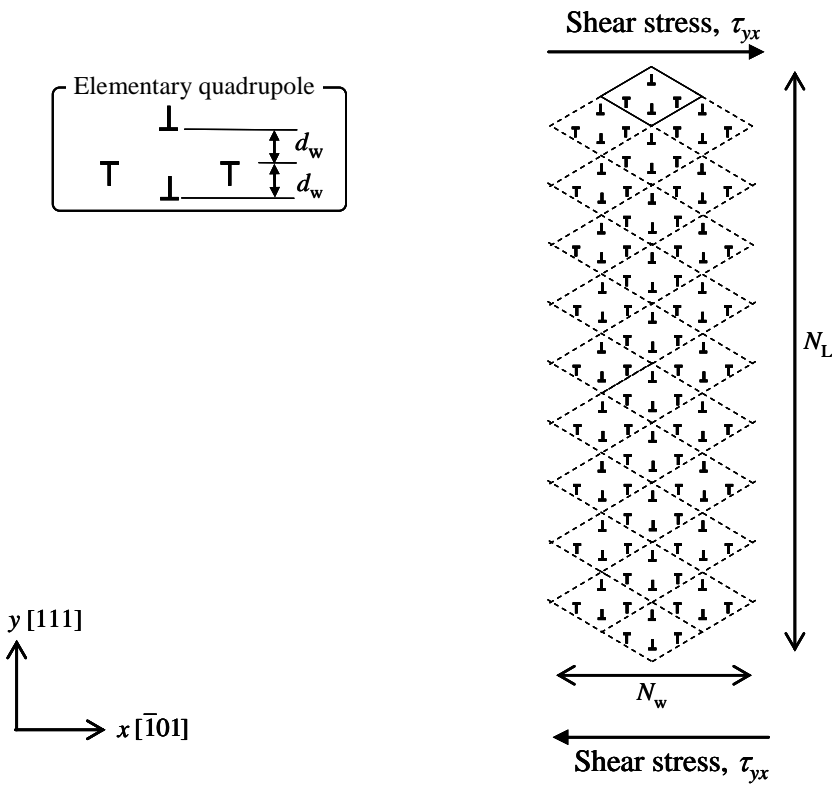

Fig. 2 Simulation models of (a) veins and (b) dislocation walls. $\perp$ and $\mathrm{T}$ denote positive and negative edge dislocations, respectively.

Figure 2 shows simulation models of veins and dislocation walls with Taylor-Nabarro lattices in a copper single crystal. The edge dislocations are in the primary slip plane of (111) and the $x$-axis corresponds to the primary slip direction of [101] and the $y$-axis is along the [111] direction of the crystal. For copper, the magnitude of Burger vector $b=0.25 \mathrm{~nm}$, the shear modulus $G=41.05 \mathrm{GPa}$, the Poisson 
ratio $v=0.33$, and the cut-off radius of integration path $R_{c}=1.0 \mu \mathrm{m}$ are used in this study $[22,23]$. Taylor-Nabarro lattices of the vein structure consist of the elementary quadrupole, as shown by the solid lines in Fig. 2(a), and the simulation model of the vein structure has $N_{\mathrm{v}} \times N_{\mathrm{v}}$ quadrupoles with $d_{\mathrm{v}}=15 \mathrm{~nm}$. Simulation models with $N_{\mathrm{v}}=3$, 9, and 15 are analyzed in this study. The simulation model of a dislocation wall consisting of elementary quadrupoles illustrated in Fig. 2(b) has $N_{\mathrm{w}}$ quadrupoles in the $x$-direction and $N_{\mathrm{L}}=10$ quadrupoles in the $y$-direction. The distance between slip planes of neighboring dislocations, $d_{\mathrm{w}}$, is same as that for veins, i.e., $d_{\mathrm{w}}=15 \mathrm{~nm}$. Dislocation walls with $N_{\mathrm{w}}=1$ and 3 are investigated.

\subsection{Stable structure}

At first, the dislocation structures shown in Figs. 2(a) and (b) are relaxed by discrete dislocation dynamics (DDD) [24-26] calculations under the applied uniform shear stress. During the relaxation of dislocation structures, the dislocation motion is determined by the experimentally obtained velocity equation for the copper single crystal

$$
v_{i}=v^{*} \zeta_{i} \frac{\tau_{i}}{\left|\tau_{i}\right|}\left(\frac{\left|\tau_{i}\right|}{\tau^{*}}\right)^{m}
$$

where $v^{*}=1.0 \times 10^{-2} \mathrm{~m} / \mathrm{s}, \tau^{*}=0.25 \times 10^{3} \mathrm{~Pa}$, and $m=0.7$ [27]. Numerical integration is performed by the Euler method and the time step is taken to be $\Delta t=1.0 \mathrm{ps}$. Here, $v_{i}$ is the velocity of the $i$-th dislocation and $\tau_{i}$ is the shear stress acting on the $i$-th dislocation, which is calculated by the superposition of the external load induced shear stress $\tau_{y x}$ and other dislocations induced stress fields,

$$
\tau_{i}=\tau_{y x}+\frac{G}{2 \pi(1-v)} \times \sum_{j \neq i} \zeta_{j} b \frac{\left(x_{i}-x_{j}\right)\left\{\left(x_{i}-x_{j}\right)^{2}-\left(y_{i}-y_{j}\right)^{2}\right\}}{\left\{\left(x_{i}-x_{j}\right)^{2}+\left(y_{i}-y_{j}\right)^{2}\right\}^{2}} .
$$


To obtain the fully relaxed equilibrated dislocation structure, the DDD calculation is performed until all the Peach-Koehler forces acting on the dislocations are less than $2.5 \times 10^{-7} \mathrm{~N} / \mathrm{m}$. The increment, $\Delta \tau_{y x}$, and the relaxation are repeated until the onset of collapse of dislocation structure.

\subsection{Vein structure}

Figure 3 shows the variation of the total energy per dislocation, $\Pi / 4 N_{\mathrm{v}}^{2}$, for veins with $N_{\mathrm{v}}=3,9$, and 15 as a function of the applied shear stress, $\tau_{y x}$. The total energy increases smoothly with the applied shear stress. At a high shear stress level $\left(\tau_{y x}>15\right.$ MPa in $N_{\mathrm{v}}=9$ ), the slope of the energy curve becomes larger, and the total energy increases rapidly at the last.

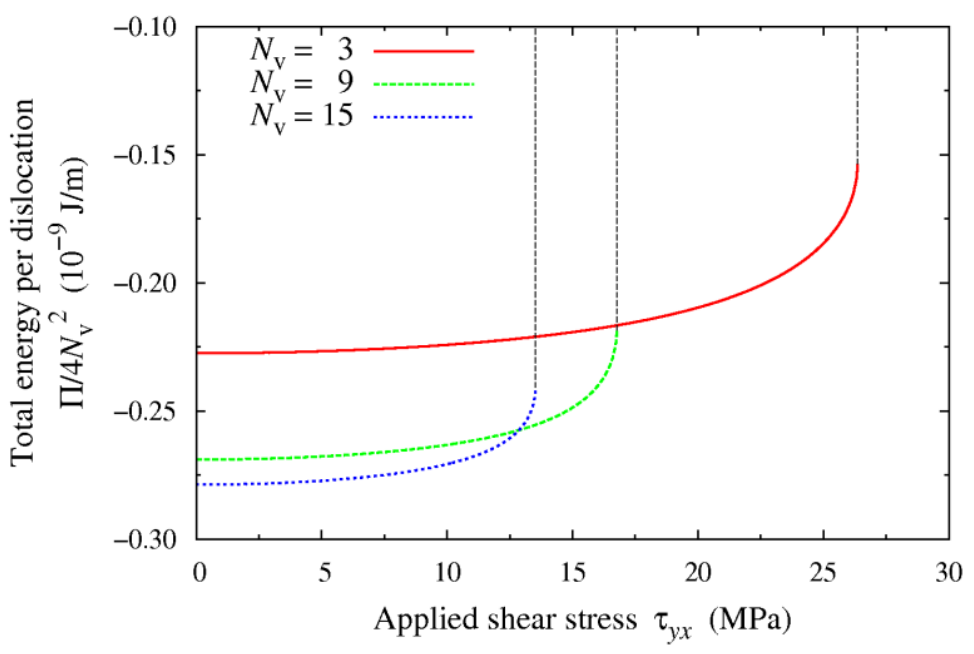

Fig. 3 Total energy per dislocation, $\Pi / 4 N_{\mathrm{v}}^{2}$, of veins with $N_{\mathrm{v}}=3,9$, and 15 as a function of applied shear stress $\tau_{y x}$.

Figures 4(a) and (b) show snapshots of a vein with $N_{\mathrm{v}}=9$ immediately before $\left(\tau_{y x}=\right.$ 16.763 $\mathrm{MPa})$ and during $\left(\tau_{y x}=16.764 \mathrm{MPa}\right)$ the rapid increase of the total energy, respectively. For a shear stress of $\tau_{y x}=16.763 \mathrm{MPa}$, the rhomboidal shape of the vein is preserved, although the vein structure is stretched in the $x$-direction relative to the 
unloaded vein. On the other hand, for $\tau_{y x}=16.764 \mathrm{MPa}$, the vein structure breaks into dipolar walls of dislocations and the dislocation walls move away from each other. The dislocation wall at the right tip of the vein structure exhibits an eminent displacement than the others. Therefore, the rapid increase of total energy indicates the collapse of vein structure. Veins with $N_{\mathrm{v}}=3$ and $N_{\mathrm{v}}=15$ exhibit similar collapse behaviors with the applied shear stress. Table 1 lists the critical shear stresses for veins with $N_{\mathrm{v}}=3,9$, and 15. The critical shear stress is higher for a smaller vein, which means that the smaller vein structure is more resistant to external load.

(a) Before instability $\left(\tau_{y x}=16.763 \mathrm{MPa}\right)$

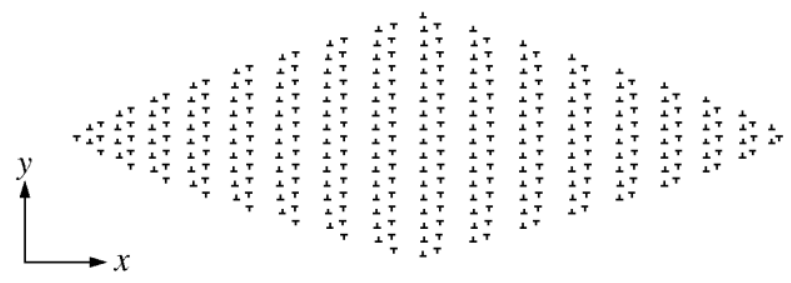

(b) During instability $\left(\tau_{y x}=16.764 \mathrm{MPa}\right)$

(i) $t=3.45 \mu \mathrm{s}$

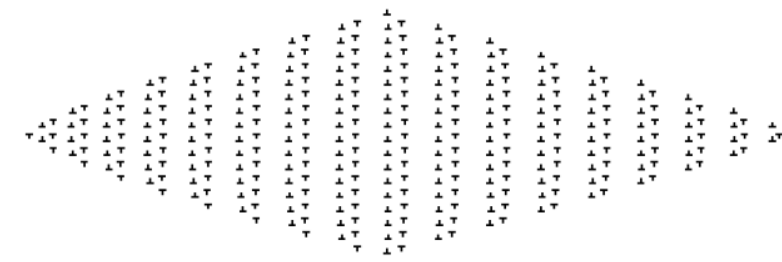

(ii) $t=3.46 \mu \mathrm{s}$

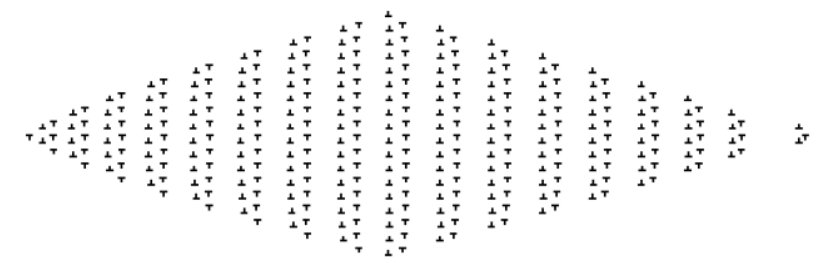

(iii) $t=3.47 \mu \mathrm{s}$

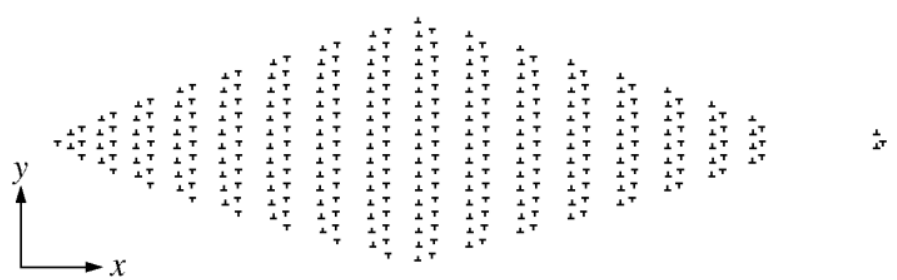

Fig. 4 Snapshots of a vein with $N_{\mathrm{v}}=9$ (a) before and (b) during the instability. 
Table 1 Critical shear stress, $\tau_{\mathrm{c}}$, of veins with $N_{\mathrm{v}}=3,9$, and 15 .

\begin{tabular}{lccc}
\hline \hline$N_{\mathrm{v}}$ & 3 & 9 & 15 \\
\hline$\tau_{\mathrm{c}}(\mathrm{MPa})$ & 26.360 & 16.764 & 13.532 \\
\hline \hline
\end{tabular}

Figure 5 shows the relationships between the minimum eigenvalue $\eta_{1}$ and the applied shear stress $\tau_{y x}$ for veins with $N_{\mathrm{v}}=3,9$, and 15 . Here, $\eta_{1}$ is normalized by its magnitude in the unloaded state, $\eta_{1}^{(0)}$. The arrows in Fig. 5 indicate the critical shear stresses of the models, $\tau_{\mathrm{c}}$. For the vein with $N_{\mathrm{v}}=9$, the magnitude of $\eta_{1}$ continuously decreases with the applied shear stress and finally reaches zero at $\tau_{y x}=16.764 \mathrm{MPa} . \eta_{1}$ $=0$ corresponds exactly with the instability condition. In addition, for veins with $N_{\mathrm{v}}=3$ and $15, \eta_{1}=0$ also does. This indicates that the collapse of the vein structure is caused by the structural instability of dislocations. Moreover, since the minimum eigenvalue $\eta_{1}$ decreases monotonically as the applied shear stress approaches the critical stress (the onset of instability), it is possible to evaluate the stable degree of the structure with the reduction of $\eta_{1}$. However, discussion on this issue is beyond the scope of this study and remains for further investigation.

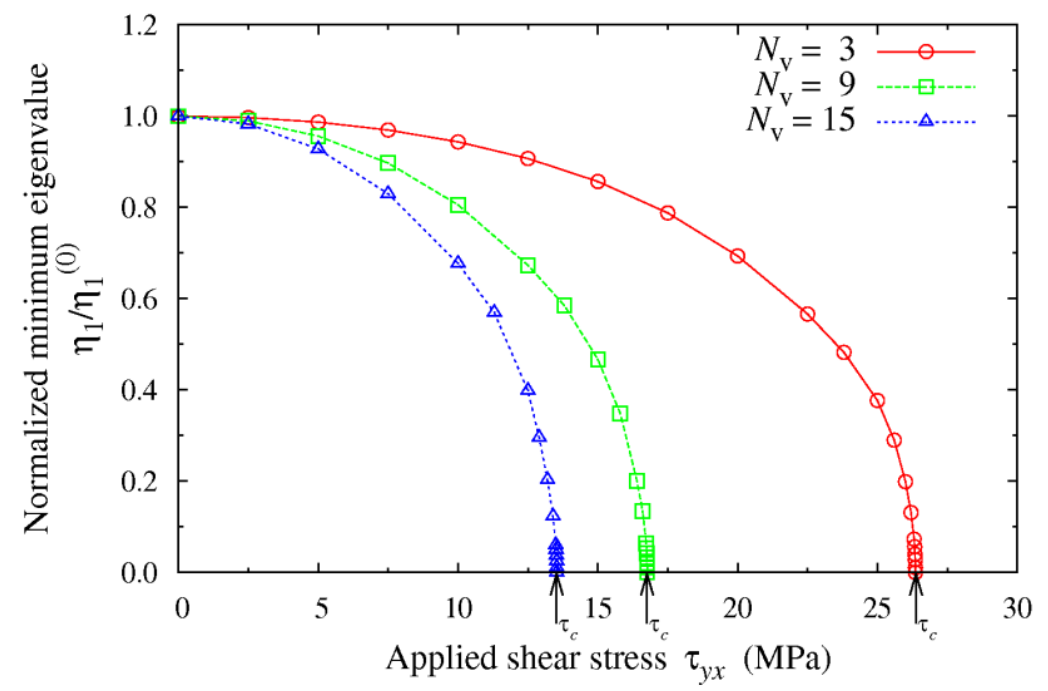

Fig. 5 Relationships between the normalized minimum eigenvalue, $\eta_{1} / \eta_{1}^{0}$, and applied shear stress $\tau_{y x}$. Arrows indicate the critical shear stress $\tau_{c}$. 
Figure 6 shows the eigenvector of the $N_{\mathrm{v}}=9$ vein at the onset of instability $\left(\eta_{1}=0\right)$, i.e., the instability mode at the collapse of vein. The instability mode vector reveals that the vein decomposes into dislocation walls and the walls tend to depart from each other. In addition, a prominent displacement exists at the right tip of the vein structures. Therefore, the instability mode represented by the eigenvector $\boldsymbol{p}_{1}$ correctly describes the dislocation motions at the onset of collapse.

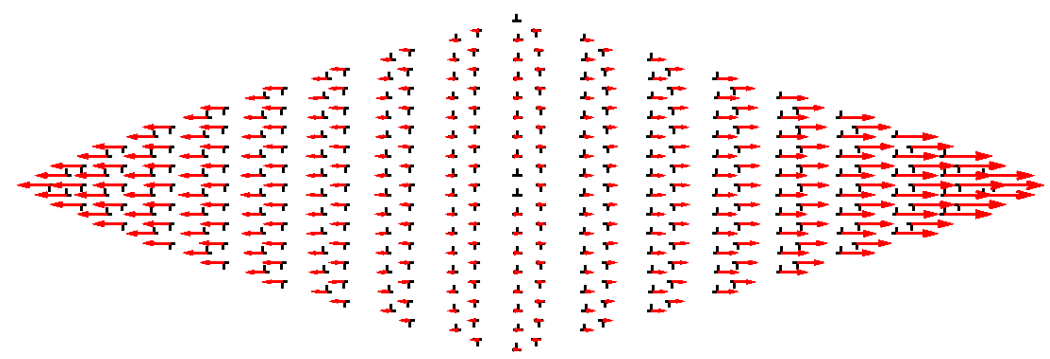

Fig. 6 Instability mode vector of the vein with $N_{\mathrm{v}}=9$.

\subsection{Dislocation wall}

Figure 7 shows the relationships between the total energy per dislocation, $\Pi / 4 N_{\mathrm{v}}^{2}$, of the dislocation walls with $N_{\mathrm{w}}=1$ and 3 and the applied shear stress, $\tau_{y x}$. The total energies of dislocation walls of both sizes increase smoothly with the applied shear stress, and rapidly increase at a specific stress. This is the same trend as that observed for veins, as discussed in the previous section. 


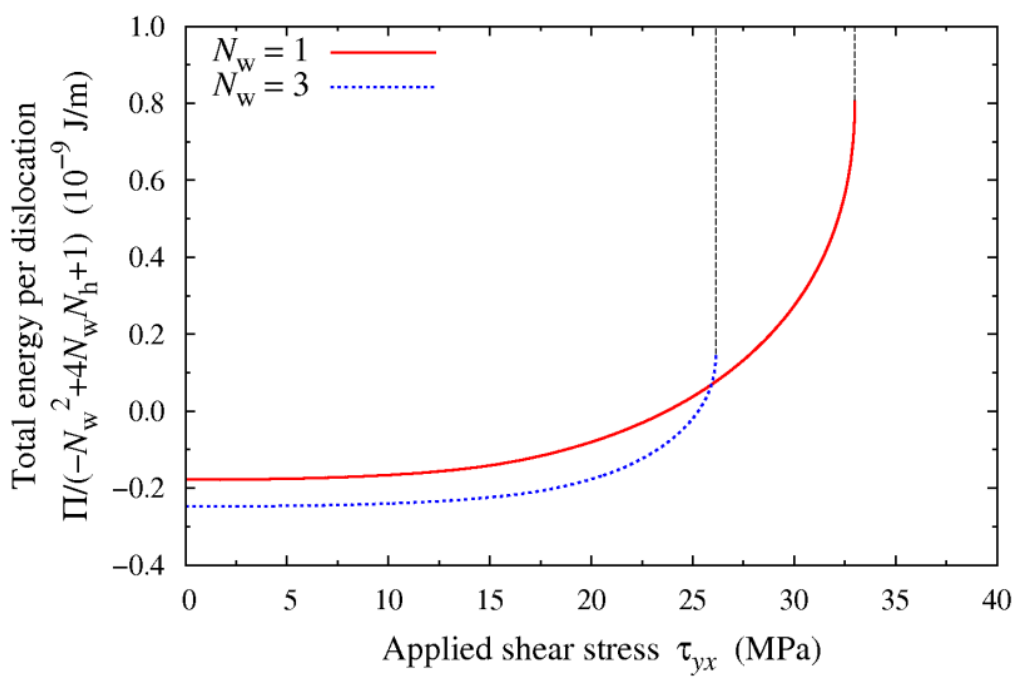

Fig. 7 Total energy per dislocation, $\Pi / 4 N_{\mathrm{v}}^{2}$, of dislocation walls with $N_{\mathrm{w}}=1$ and 3 as a function of applied shear stress $\tau_{y x}$.

Figures 8(a) and (b) show snapshots of the $N_{\mathrm{w}}=3$ dislocation wall immediately before $\left(\tau_{y x}=26.150 \mathrm{MPa}\right)$ and during $\left(\tau_{y x}=26.151 \mathrm{MPa}\right)$ the rapid increase of the total energy, respectively. At a shear stress of $\tau_{y x}=26.150 \mathrm{MPa}$, although the dislocation wall is broader than its unloaded configuration, the wall structure is still kept. However, at $\tau_{y x}$ $=26.151 \mathrm{MPa}$, the quadrupolar dislocation wall decomposes into dipolar dislocation walls and a single dislocation array, which show the trend of leaving each other. Thus, the dislocation wall collapses during the rapid increase in the total energy. Table 2 lists the critical shear stresses $\tau_{\mathrm{c}}$ for dislocation walls with $N_{\mathrm{w}}=1$ and 3 . The critical shear stress of smaller dislocation wall is larger, which indicates the higher instability. 
(a) Before instability $\left(\tau_{y x}=26.150 \mathrm{MPa}\right)$

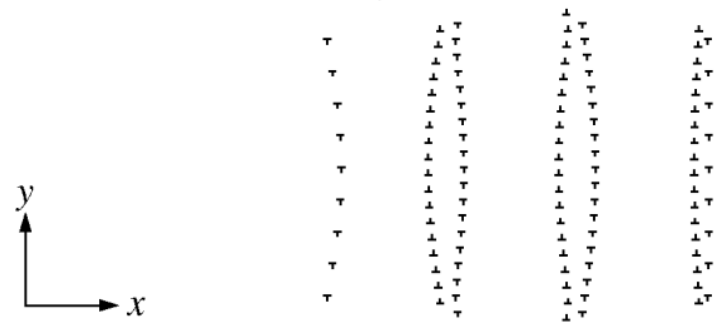

(b) During instability ( $\left.\tau_{y x}=26.151 \mathrm{MPa}\right)$

(i) $t=0.52 \mu \mathrm{s}$

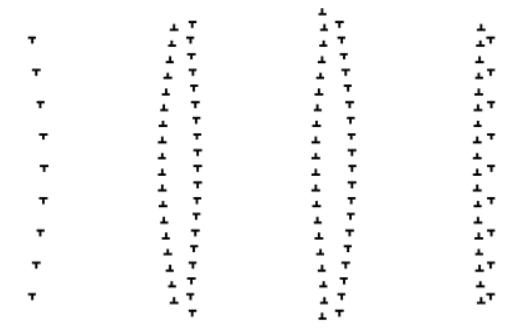

(ii) $t=0.55 \mu \mathrm{s}$

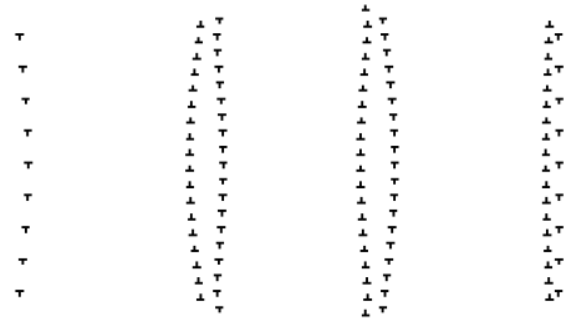

(iii) $t=0.58 \mu \mathrm{s}$
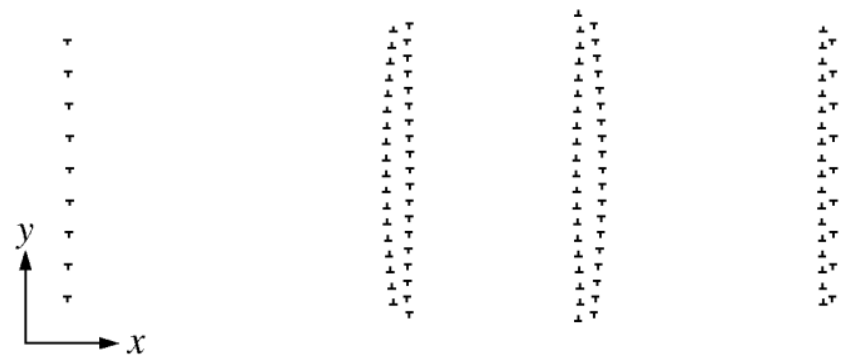

Fig. 8 Snapshots of dislocation wall with $N_{\mathrm{w}}=3$ (a) before and (b) during the instability.

Table 2 Critical shear stress, $\tau_{\mathrm{c}}$, of dislocation walls with $N_{\mathrm{w}}=1$ and 3 .

\begin{tabular}{lcc}
\hline$N_{\mathrm{w}}$ & 1 & 3 \\
\hline$\tau_{\mathrm{c}}(\mathrm{MPa})$ & 32.995 & 26.151 \\
\hline \hline
\end{tabular}


Figure 9 shows the relationships between the minimum eigenvalue, $\eta_{1}$, and the applied shear stress, $\tau_{y x}$, of dislocation walls with $N_{\mathrm{w}}=1$ and 3 . Similar to veins, the minimum eigenvalue continuously decreases with increasing applied shear stress. All the minimum eigenvalues of the dislocation walls finally obtain zero, $\eta_{1}=0$, at the critical shear stresses, which means that $\eta_{1}=0$ exactly represents the critical condition for instability.

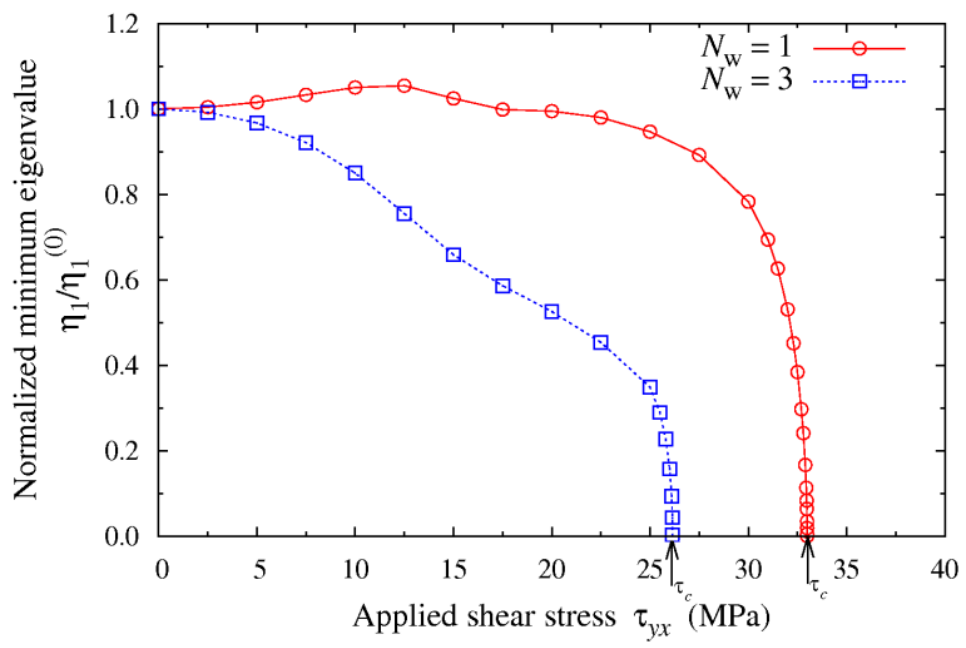

Fig. 9 Relationships between normalized minimum eigenvalue, $\eta_{1} / \eta_{1}^{0}$, and applied shear stress $\tau_{y x}$. Arrows indicate the critical shear stresses, $\tau_{c}$.

Figure 10 shows the instability mode vector $\boldsymbol{p}_{1}$ of the $N_{\mathrm{w}}=3$ dislocation wall at the onset of instability. It shows that the quadrupolar dislocation wall breaks into dipolar walls and a single dislocation array, which induces the collapse of the quadrupolar dislocation wall. This is identical to the displacement behavior of dislocations at the onset of collapse. Thus, the proposed criterion precisely describes the instability of dislocation walls. 


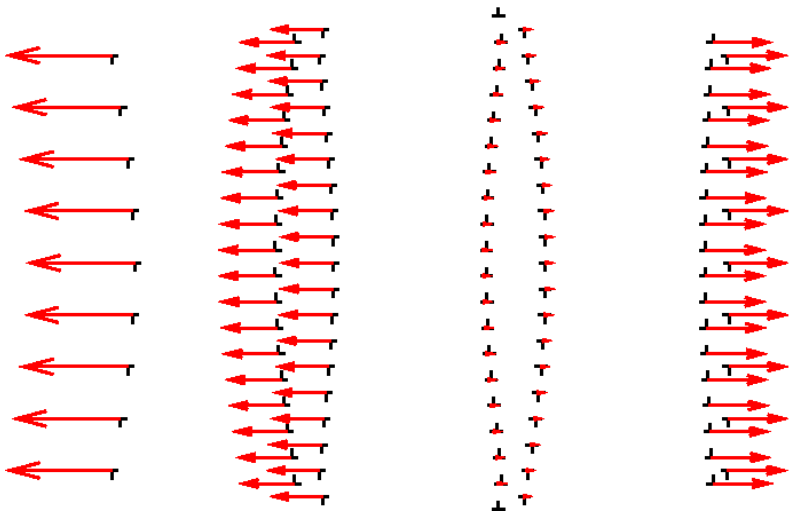

Fig. 10 Instability mode vector of dislocation wall with $N_{\mathrm{w}}=3$.

In summary, the mechanical instability criterion proposed in this study correctly describes the onset of instability and the corresponding displacement mode at the onset of collapse for different dislocation structures (i.e., veins and dislocation walls) of different sizes. Owing to this success, we can address the mechanical instability of more complex dislocation structures such as PSBs by using the proposed criterion.

\section{Conclusion}

In this study, to investigate the mechanical instability of arbitrary dislocation structures, a criterion of mechanical instability is proposed to exactly evaluate the critical condition for the onset of instability and the instability mode at the collapse of dislocation structures. The developed criterion is applied to veins and dislocation walls under external loads. The obtained results are summarized as follows:

(1). The instability criterion is evaluated by the positiveness of the minimum eigenvalue, $\eta_{1}$, of Hessian matrix, $\mathbf{H}$, whose components are second-order differentials of the potential energy of the system with respect to the dislocation coordinates. The displacement mode at the onset of collapse of dislocation structures (the instability mode) is represented by the eigenvector of the matrix at $\eta_{1}=0$.

(2). The proposed criterion is applied to a loaded vein structure, and the instability of 
the vein is successfully picked up by the criterion for models with different sizes. The instability mode represented by the eigenvector, $\boldsymbol{p}_{1}$, accurately describes the displacement mode at the collapse of the vein.

(3). For dislocation walls, the critical condition for the onset of instability and the corresponding displacement mode at collapse are precisely determined by the proposed criterion.

(4). The successful application of the proposed criterion to veins and dislocation walls under external loads indicates that the criterion is a powerful tool for investigating the instability of arbitrary dislocation structures, and it enable us to address the mechanical instability of more complex dislocation structures such as PSBs.

\section{Acknowledgment}

This study was supported by a Grant-in-Aid for Scientific Research (S) (Grant No. 21226005) of the Japan Society of the Promotion of Science (JSPS).

\section{References}

[1] J. Friedel, Dislocations, Pergamon, Oxford, 1967.

[2] J.M. Finney, C. Laird, Philos. Mag. A 31 (1975) 339-366.

[3] U. Essmann, U. Gosele, H. Mughrabi, Philos. Mag. A 44 (1981) 405-426.

[4] P.R. Swann, in: G. Thomas, J. Washburn (Eds.), Electron Microscopy and Strength of Crystals, Interscience, New York, 1963, pp.131.

[5] A.S. Keh, S. Weissman, in: G. Thomas, J. Washburn (Eds.), Electron Microscopy and Strength of Crystals, Interscience, New York, 1963, pp.231.

[6] S. Mader, A. Seeger, H.M. Thieringer, J. Appl. Phys. 34 (1963) 3376-3386.

[7] H. Mughrabi, Mat. Sci. Eng. 85 (1987) 15-31. 
[8] M.S. Whelan, in: P.B. Hirsch (Ed.), The Physics of Metals, Part 2: Defects, Cambridge University, London, 1975, pp. 98.

[9] U. Essmann, H. Mughrabi, Philos. Mag. A 40 (1979) 731-756.

[10] P.J. Woods, Philos. Mag. 28 (1973) 155-191.

[11] N. Hansen, D. Kuhlmann-Wilsdorf, Mat. Sci. Eng. 81 (1986) 141-161.

[12] D. Kuhlmann-Wilsdorf, Mater. Res. Innov. 1 (1989) 265-296.

[13] S.J. Basinski, Z.S. Basinski, A. Howie, Philos. Mag. 19 (1969) 899-924.

[14] N. Thompson, N.J. Wadsworth, N. Louat, Philos. Mag. 1 (1956) 113-126.

[15] F. Ackermann, L.P. Kubin, J. Lepinoux, H. Mughrabi, Acta Metall. 32 (1984) 715-725.

[16] P. Charsley, D. Kuhlmanm-Wilsdorf, Philos. Mag. A 44 (1981) 1351-1361.

[17] D. Kuhlmann-Wilsdorf, C. Laird, Mat. Sci. Eng. 46 (1977) 137-156.

[18] D. Kuhlmann-Wilsdorf, C. Laird, Mat. Sci. Eng. 46 (1980) 209-219.

[19] H. Suzuki, Introduction of Dislocation Theory (in Japanese), Agne, Tokyo, 1967.

[20] G.I. Taylor, Proc. Roy. Soc. London A 145 (1934) 362-368.

[21] F.R.N. Nabarro, Adv. Phys. 1 (1952) 269-395.

[22] A. Nakatani, H. Kitagawa, M. Sugizaki, Trans. JSME A 65 (1999) 575-581.

[23] E. Van der Gieseen, A. Needleman, Model. Simul. Mater. Sci. Eng. 3 (1995) 689-735.

[24] A.N. Gulluoglu, D.J. Srolovitz, R. LeSar, P.S. Lomdahl, Scripta Metall. 23 (1989) $1347-1352$.

[25] J. Lepinoux, L.P. Kubin, Scripta Metall. 29 (1987) 833-838.

[26] R.J. Amodeo, N.M. Ghoniem, Phys. Rev. B 41 (1990) 6958-6967.

[27] W.F. Greenman, T. Vreeland, D.S. Wood, J. Appl. Phys. 38 (1967) 3595-3603. 